\title{
Connectivity and Creativity in Semantic Neural Networks
}

\author{
Nagendra Marupaka and Ali A. Minai, Senior Member, IEEE
}

\begin{abstract}
Creativity and insight are distinctive attributes of human cognition, but their neural basis remains poorly understood due to the difficulty of experimental study. As such, computational modeling can play an important role in understanding these phenomena. Some researchers have proposed that creative individuals have a "deeper" organization of knowledge, allowing them to connect remote associates and form novel ideas. It is reasonable to assume that the depth and richness of semantic organization in individual minds is related to the connectivity of neural networks involved in semantic representation. In this paper, we use a simple and plausible neurodynamical model of semantic networks to study how the connectivity structure of these networks relates to the richness of the semantic constructs, or ideas, they can generate. This work is motivated, in part, by research showing that experimentally obtained semantic networks have a specific connectivity pattern that is both small-world and scalefree. We show that neural semantic networks reflecting this structure have richer semantic dynamics than those with other connectivity structures. Though simple, this model may provide insight into the important issue of how the physical structure of the brain determines one of the most profound features of the human mind - its capacity for creative thought.
\end{abstract}

\section{INTRODUCTION}

Creativity and insight are often seen as arising from the ability to connect disparate concepts or ideas, i.e, to make unexpected connections in semantic space [1], [2], [3], [4], [5], [6]. The experience of unusual combinations leading to creativity has been documented by several scientists. For example, Einstein is quoted as saying, "Taken from a psychological viewpoint ... combinatory play seems to be the essential feature in productive thought - before there is any connection with logical construction in words or other kinds of signs which can be communicated to others" (quoted in [2], [4]). Similarly, Poincare, described his own creative thinking process as follows: "Ideas arose in crowds; I felt them collide until pairs interlocked, so to speak, making a stable combination. ... the only combinations that have a chance of forming are those where at least one of the elements is one of those atoms freely chosen by our will. Now, it is evidently among these that is found what I called the good combination. ... among the great numbers of combinations blindly formed almost all are without interest and without utility" [7] (quoted in [4]). The latter quote

Nagendra Marupaka is with the School of Electronic and Computer Systems, University of Cincinnati, Cincinnati OH 45221, Email: marupana@mail.uc.edu. Ali Minai is with the School of Electronic and Computer Systems, University of Cincinnati, Cincinnati OH 45221, Email: Ali.Minai@uc.edu

Acknowledgement: This work was supported in part by a National Science Foundation Human and Social Dynamics Program grant to Ali Minai (BCS-0728413), which includes support from the Deputy Director of National Intelligence, and by a National Science Foundation CreativeIT grant to Ali Minai (IIS-0855714) points to both the utility and dangers of unusual combinations - most combinations generated by the creative mind are not useful, or even nonsensical, but this is the price for discovering the rare combination of uncommon value.

Since conceptual combinations arise in the minds of individuals, they must be the product of the cognitive dynamics of these individual minds. The key idea is that creativity depends on how associations between concepts are organized in the mind. Presumably, individuals whose minds link concepts that most people do not are likelier to find them when needed. This idea was formalized by Mednick [2] as an associative hierarchy, which relates the uniqueness (or "unusualness") of associations in the individual's mind with the strength of these associations. He argued that non-creative individuals (or individuals thinking in contexts where they are non-creative) have a steep associative hierarchy, where common associations are very strong, but uncommon ones are much weaker. Such an individual is likely to think mainly in terms of common associations and generate conventional, non-creative ideas. The creative individual, in contrast, has a flatter association hierarchy where even unusual concepts are fairly strong, and therefore likelier to be discovered during associative search.

Another important factor in creativity is the problem of fixation - the inability to break out of conventional thinking or standard practices. This often occurs in individuals with high expertise in narrow domains because the associations underlying their standard methods are much stronger than those that would lead to non-standard ones. Faced with a particular task, such individuals are likelier to keep returning to the same standard ideas. One interesting aspect of Mednick's hypothesis is that creative individuals may be less likely to generate conventional combinations than noncreative ones, thus avoiding fixation. It has been argued that individuals with broad but relatively shallow expertise may be more creative than those with deep and narrow expertise [6]. Indeed, experiments have shown that the inclusion of a few unconventional thinkers can enhance the creativity of a whole group even if these thinkers are not especially knowledgeable [8], [9].

Given the significance of how conceptual associations are organized, it is important to ask three questions:

1) What is the actual organization of associations is in the minds of individuals?

2) How do these associations generate a "train of thought" that may lead to creativity?

3) Are some types of associative organization more congenial to creative thinking than others?

The focus of this paper is on the last question. 


\section{BACKGROUND AND MOTIVATION}

The organization of associative memory has been studied very widely, typically using word association or cued recall tests [10], [11], [12], [13], [14]. Such studies have generated a rich body of data on association norms, which have been used to develop theories of associative recall (e.g., [11], [12], [15], [13]). Very interesting results have emerged from a network analysis of word association data by Steyvers and Tenenbaum [16], who showed that such associative networks possess two interesting properties: 1) A small-world architecture; and 2) A power-law degree distribution. They also proposed a model of semantic evolution that could produce such networks, and have argued that these properties might be representative of associative semantic networks in general. While these networks represent data averaged over a large number of test subjects, they provide a good starting point for investigating the relationship between associative network structure and creativity. In particular, one may ask how the two characteristics found by Steyvers and Tenenbaum impact the search for conceptual combinations separately and jointly.

The degree distribution of complex networks has recently been a subject of great interest, with particular attention focusing on networks with power-law degree distribution [17], [18], i.e., $p(k) \sim k^{\beta}$, where $p(k)$ is the probability of a node having $k$ connections. Random networks with homogeneous uniform probability of connection between all node pairs are expected to have a Poisson degree distribution with a pronounced mode and an exponential tail, unlike the "fat tail" of the power-law distribution. Networks with the latter degree distribution are called scale-free networks, and are known to be generated by processes of preferential attachment, where new nodes join the network by connecting preferentially to already well-connected nodes [17]. This results in the emergence of hub nodes with very high connectivity, which lie in the fat tail of the degree distribution. It has been proposed that many real-world networks, including the Internet and the World-Wide Web, are scale-free [19], [20], [21].

Small-world networks [22], [23] are networks with a high degree of clustering, $C$, among nodes as well as low mean shortest path length, $D$, between node pairs. Clustering is defined as the mean probability that the neighbors of two directly connected nodes are also directly connected. Networks with uniform random connectivity typically have low $D$ and low $C$, whereas networks with local connectivity have high $D$ and $C$. Thus, small-world networks, with low $D$ and high $C$, represent a distinct class of networks, and have been found to be good models for many types of real-world complex networks such as power grids, neural networks and social networks [22], [21]. It has been shown that neural networks with small world structure are very efficient as associative memories [24], and small world characteristics have been found in cortical neural networks [25]. It has been suggested that small-world connectivity in the brain may underlie high creativity in individuals [6].

Scale-free networks have low $D$, but not necessarily low
$C$, while small world networks do not necessarily have a power law degree distribution. Thus, the networks found by Steyvers and Tenenbaum represent a special subclass of networks lying in the intersection of the two sets.

In this paper, we describe a simple but neurally plausible computational model for associative search through the space of conceptual combinations in semantic neural networks, and use it to compare the characteristics of the resulting search in networks with four different types of connectivity: Random (RA), Localized (LO); small-world (SW); and SteyversTenenbaum (ST). In particular, we consider the following issues:

1) Do networks with distinct connectivity differ significantly in the richness of their search through the space of conceptual combinations?

2) Does the ST model provide any advantage in terms of the search for conceptual combinations, and if so, does this arise from the small-world property, the scale-free property, or any from a combination of both?

It is important to emphasize that our work differs nominally from most models of associative recall (e.g., [15]) in two ways: 1) We consider the recall of conceptual combinations rather than single associates; and 2) We consider free recall (or spontaneous thinking) rather than cued recall. However, insofar as conceptual combinations can themselves be seen as more complex concepts, our model can be considered as implementing distributed form of associative chaining.

\section{MODEL DESCRIPTION}

\section{A. Network Model}

The neural semantic network model we use is an extension of one we have recently developed as part of a larger model of cognitive function [26], [27], [28]. It is based on the concept of competitive itinerant dynamics, as described below.

The semantic network is modeled as a network of $N$ concept units, each representing one concept. Each unit can be seen as a neural assembly tuned to a particular concept. Since our purpose in this paper is just to study the dynamics of networks with various types of connectivity, we use abstract concepts instead of actual words. The connections between units represent associations, with the connection from unit $j$ to unit $i$ denoted by $w_{i j}$. For simplicity, we assume that the connections are binary and symmetric, i.e., either two concepts are mutually associated with a weight of 1 or not associated, with a weight of 0 . The output of unit $i$ at time $t$ is denoted by $x_{i}(t)$, and the net input to a unit $i$ and time $t$ is given by:

$$
x_{i}(t)=\sum_{j=1}^{N} w_{i j}(t) x_{j}(t)+\gamma_{n o i s e} \xi_{i}(t)
$$

where $x_{j}$ are the outputs from units $j, w_{i j}$ are binary weights to concept unit $i$ from concept unit $j, \xi_{i}(t)$ is uniform 
white noise between 0 and 1 , and $\gamma_{\text {noise }}$, is a fixed gain parameter.

The state of concept unit $i$ at time $t$ is given by:

$$
y_{j}(t)=\alpha y_{j}(t-1)+(1-\alpha) x_{i}(t)
$$

where $\alpha$ is an inertial parameter.

Activity in the network is competitive, and the $K$ nonrefractory units with the highest $y(t)>0$ are allowed to fire at time $t$. The output of unit $i$ is calculated as:

$$
x_{i}(t)=f\left(y_{i}(t)\right)= \begin{cases}1, & \text { if } y_{i}(t) \in\{k \text { most excited units }\} \\ 0, & \text { otherwise }\end{cases}
$$

Unit activity and excitability are modulated by two other processes: Refractoriness and synaptic modulation.

Refractoriness: Once fired, unit $i$ may remain active for an activity duration $\varphi$ if $y_{i}^{c}(t)$ remains sufficiently high, after which it enters a refractory period. This is modeled through a resource, $r_{i}(t) \in R(t)$, with the following dynamics:

$$
r_{i}(t)= \begin{cases}\left(1-\lambda_{-}\right) r_{i}(t-1), & \text { if active } \\ r_{i}(t-1)+\lambda_{+}\left(1-r_{i}(t-1)\right), & \text { if inactive }\end{cases}
$$

where $\lambda_{-}$is the resource depletion rate, and $\lambda_{+}$is the resource recovery rate. A neuron is said to be in a refractory state if:

$$
r_{i}(t) \leq \Theta_{r}
$$

Thus, a unit's resource is depleted when it fires and recovers when it is inactive (due to lack of stimulus or refractoriness).

Synaptic Modulation: Synapses that are excited repeatedly by pre-synaptic activity temporarily become habituated to it and diminish in strength while the activity persists, and then recover gradually when activity ceases. This is modeled as follows:

$$
w_{i j}(t)= \begin{cases}\left(1-\psi_{-}\right) w_{i j}(t-1), & \text { if active } \\ w_{i j}(t-1)+\psi_{+}\left[w_{i j 0}-w_{i j}\right] & \text { if inactive }\end{cases}
$$

where $\psi_{\text {- and }} \psi_{+}$represent the synaptic decay and recovery rates respectively and $w_{i j o}$ represents the initial weight of the synapse. Synaptic modulation has recently been proposed as an important component of neural information processing and short-term memory [29], [30].

\section{B. Network Connectivity Generation}

Since the Steyvers-Tenenbaum model is the focus, we first generate that network, and then generate the other three networks to be comparable to it. All connections in the networks are undirected, resulting in symmetric connectivity. The networks are generated as follows:

Steyvers-Tenenbaum (ST) Network: This network is generated according to the method described by Steyvers and Tenenbaum in [16]. The network begins with $M$ fully interconnected nodes, followed by the addition of $N-M$

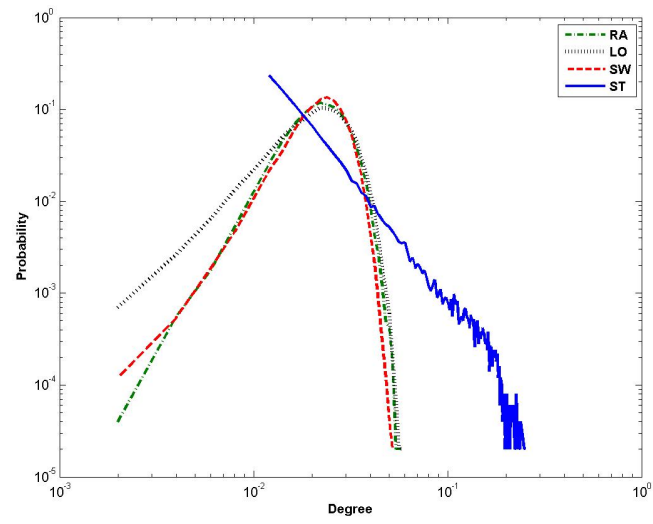

Fig. 1. Degree distribution for the four networks

nodes one at a time. Each new node is added in two steps: 1) An existing node, $i$, is chosen with probability $P_{i}=$ $k_{i} / \sum_{j} k_{j}$, where $k_{j}$ is the degree of node $j$; 2) The new node is connected randomly to $M$ other nodes from the set of nodes to which $i$ is already connected. Thus, the new node can be seen as a partial replication of node $i$, which is chosen with a preference for higher degree nodes. The resulting network is both small-world and scale-free, with an exponent near 3. For the simulations described here, we use $N=500$ and $M=6$, giving a total of $n_{c}=2979$ bidirectional connections, so the network is extremely sparse.

Random (RA) Networks: The RA network comprises $N$ nodes with $n_{c}$ connections assigned between node pairs chosen randomly with equal probability. This results in a Poisson degree distribution for the nodes [21].

Localized (LO) Networks: For the LO network, nodes are placed randomly in 2-dimensional Euclidean space, and each node makes connections within a certain radius, $r$, of itself. The radius is chosen so that the total number of connections in the network is approximately $n_{c}$.

Small-World (SW) Networks: The SW network is obtained by rewiring short connections in the LO network randomly to more distant nodes until the mean clustering coefficient of the network matches that of the ST network.

Thus, to summarize, all nodes have the same number of nodes and connections, the ST and SW networks have the same clustering coefficient, and the LO and SW networks have the same basic connection radius.

Figure 1 shows the degree distributions for the four networks in log-log coordinates. It is clear that the RA, LO and SW networks have distributions with exponential tails while the ST network's distribution has a power law tail. It is interesting to interpret these connectivity patterns in light of Mednick's associative hierarchy. In the three networks with exponential degree distributions, each concept has approximately the same number of associations, whereas the ST network has concepts with very different numbers of associations, including some with a very large set. Thus, the associative hierarchies associated with the RA, LO and 

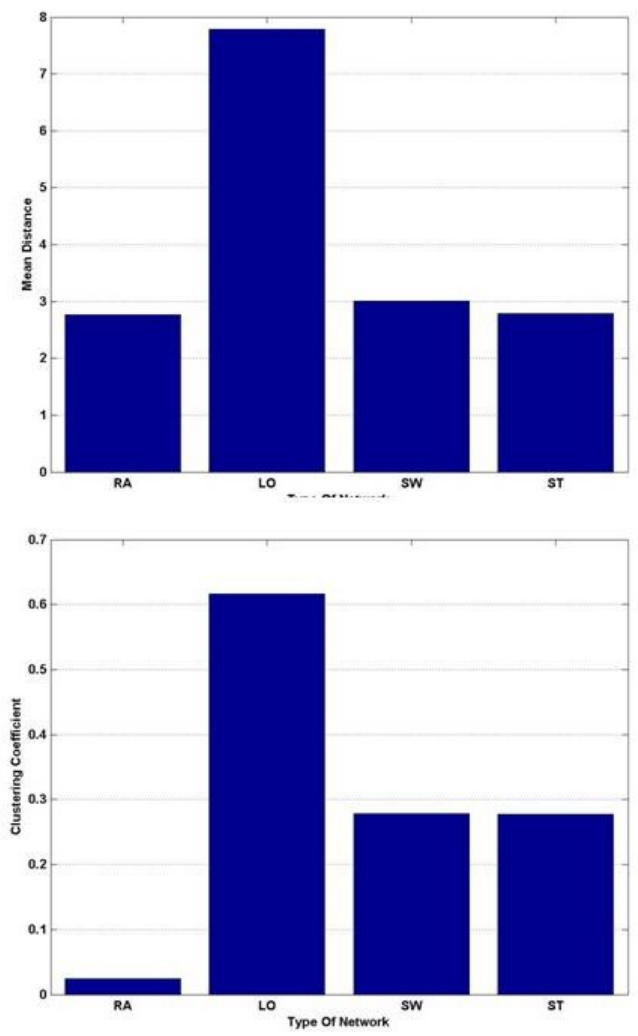

Fig. 2. Graph characteristics for the four networks: Top - mean shortest path length; Bottom - mean clustering coefficient

SW networks have a single scale and can be seen as being "shallower" than the multi-scale hierarchy associated with the ST network.

Figure 2 shows the mean shortest path length and mean clustering coefficients for the four networks. As expected, the RA network has short path length and almost no clustering, while the LO network has very long paths and very high clustering. The ST and SW networks have path lengths similar to the RA networks but much higher clustering, showing small-world characteristics [22].

\section{IDEA DYNAMICS}

As discussed earlier, we consider ideas to be conceptual combinations. In terms of the semantic network described above, an idea is represented by a set of persistently coactive concept units, or a metastable attractor. As the network activity moves through the state-space, it is itinerant [31] or "sticky", with periods of stable activity patterns punctuated by intervals of transient activity. Since each unit in the network represents a concept, any set of co-active units is a potential idea. However, we assume that only those coactive sets that persist beyond a certain duration termed the awareness threshold, $\Theta_{t h}$, are perceived consciously as ideas, whereas the rest remain subconscious. The intuition is that only units that form sufficiently strongly connected sets will be able to sustain co-activity until refractoriness or synaptic

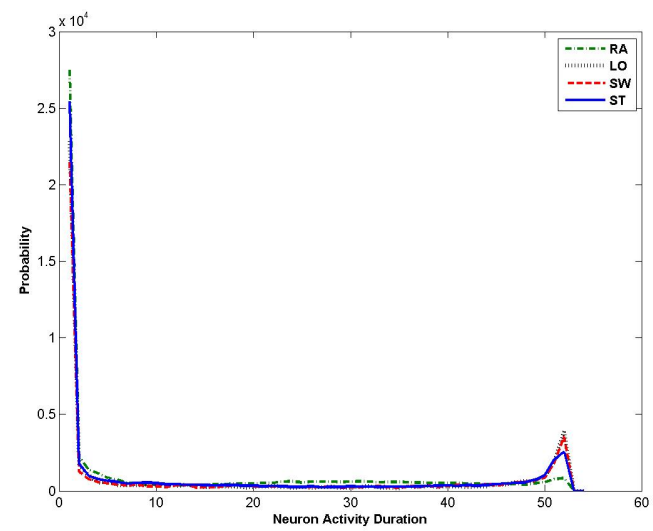

Fig. 3. Degree distribution for the four networks

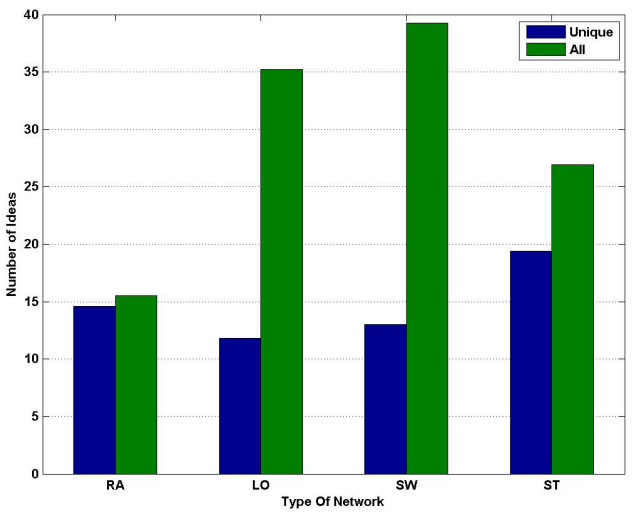

Fig. 4. Number of unique ideas produced by the four networks

modulation ends it. Such groups thus represent a metastable activity pattern - or temporary attractor - in state space. The connectivity of the network, which is defined by interconcept associations, embeds a large number of such latent ideas in the system, which are unmasked emergently by the dynamics of the system. In an intuitive sense, such attractors bring together concepts that "make sense together."

Functionally, the dynamics of the system can be seen as generating a sequence of ideas with intervening periods of transience. We characterize this functional dynamics in terms of the following attributes:

1) Productivity, $\rho$, is measured by counting the number of unique ideas generated over a finite period.

2) Efficiency, $\eta$, is a measure of how much time is "wasted" generating repeated ideas. This is calculated as:

$$
\eta=\rho / \rho_{\text {all }}
$$

where $\rho_{\text {all }}$ is the total number of ideas (including repeated ones) generated by the network. Low efficiency can be seen as a signature of fixation.

3) Coherence, $\omega$, quantifies how internally coherent the generated ideas are relative to the knowledge embed- 


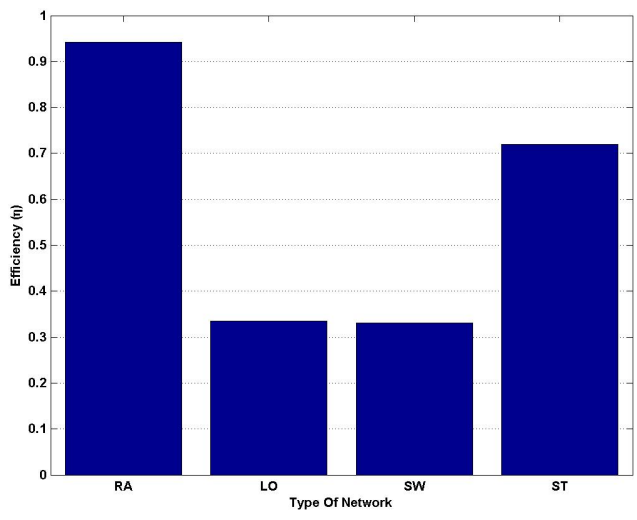

Fig. 5. Mean efficiency of the four networks

ded in the network. This is measured by calculating the clustering - or mutual connectivity - among the nodes participating in the idea. If idea $I_{k}$ has $K$ active nodes,

$$
\omega\left(I_{k}\right)=q\left(I_{k}\right) / K(K-1)
$$

where $q\left(I_{k}\right)$ is the number of connections that exist between the $K$ nodes active in $I_{k}$.

The use of cooperatively co-active groups of concepts as representations of ideas has been considered indirectly by Nelson et al. [13] in the context of implicit memory. They found that concepts that are part of such highly connected (or clustered) groups are easier to recall than those that are not.

From a neurophysiological viewpoint, a more plausible instantiation of our co-activity patterns might be in terms of emergent synchronization among neuronal assemblies, which has been suggested as the brain's main mechanism for representational binding [32], [33], [34], [35], [36], [37]. Several computational models have been developed for such systems (e.g., [38]), but we use a simpler model for clarity.

Finally, it should be noted that, while we use the term "concept" for the information represented by each network unit, they could equally well be seen as "features". The distinction between feature, concept and idea is largely a matter of position in a representational hierarchy rather than an essential difference.

\section{Simulations And Results}

Identically sized networks $(N=500)$ of all four types were simulated and evaluated in terms of the four metrics described above. The results were compared to characterize each network in terms of its semantic richness.

The first issue is to set the awareness threshold, $\Theta_{t h}$, in a meaningful way given the other dynamical parameters in the system. Figure 3 shows the distribution of how long single units remain persistently active in all four networks. This bimodal distribution indicates that, in most cases, units remain active only for a few steps before switching off. This
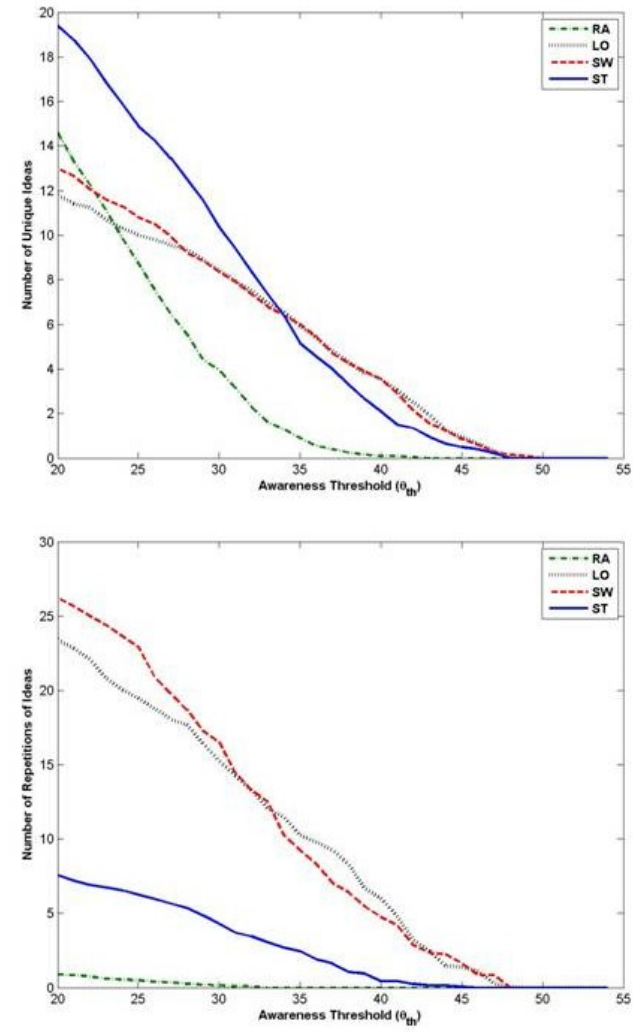

Fig. 6. Number of unique and repeated ideas generated by the four networks for various awareness thresholds

is clearly indicative of transient activity. However, in some instances, neurons remain persistently active for durations between 48 and 53 steps, which is the limit set by the resource decay rate. We hypothesize that these are the units participating in a metastable attractor - i.e., an idea - and remaining active as long as physically possible. Based on this figure, we set the awareness threshold near the lowest point of the distribution at $\Theta_{t h}=20$.

Figure 4 shows the average number of total and unique ideas generated by each network over 20 runs of 4000 time steps each. As can be seen, the LO and SW networks generate a lot of ideas, but a large fraction of these are repeated ones. In contrast, the RA network generates fewer ideas but nearly all are unique. Finally, the ST network generates the highest number of unique ideas with a relatively low level of repetitions. The useful productivity of the four networks is shown explicitly in Figure 5, which plots the efficiency, $\eta$ of each system. It shows that the RO network is the most efficient, followed by ST, with the SW and LO networks faring rather poorly.

An interesting aspect of productivity is its dependence on the awareness threshold. A larger awareness threshold makes the system more selective, with only very persistent activity patterns regarded as ideas. Figure 6 shows the number of unique and repeated ideas counted at various awareness thresholds from 20 to 54 . As can be seen, very few unique 


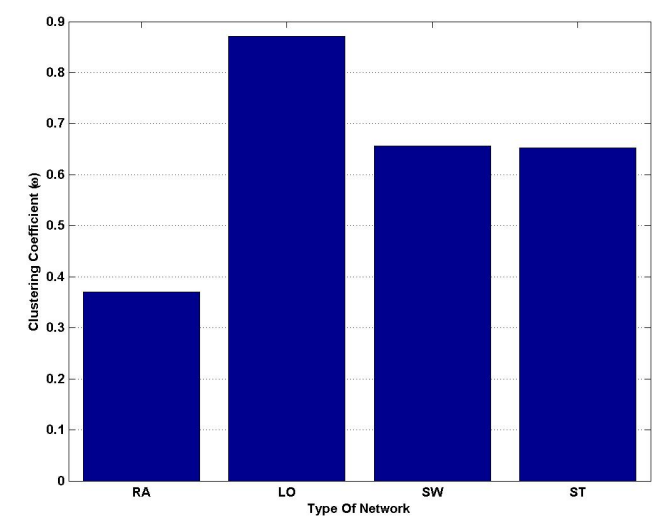

Fig. 7. Mean coherence of the unique ideas generated by the four networks

ideas are seen for high awareness thresholds. Over the range from $\Theta_{t h}=20$ to 35 , the ST network produces more unique ideas that SW or LO, whereas after that point, all three networks have similar productivity. This means that the ideas produced by the ST network last for relatively short durations compared to those generated by the LO and SW networks, again suggesting less fixation in the ST network. The SW and LO networks generally have very similar performance over most of the awareness threshold range. These networks also consistently generate more repeated ideas than the ST network.

Figure 7 shows the internal coherence of the generated unique ideas, measured as described above. We make the plausible assumption that coherence is a measure of both consistency with prior knowledge and novelty. An idea with very high coherence represents an "old" idea, since all its component concepts are already well associated with each other. In contrast, an idea with very low coherence can be seen as bordering on absurdity - a quirk of the system's dynamics. However, ideas with a moderate degree of coherence can be regarded as sensible but novel. The ability to generate moderately coherent ideas can, therefore, been seen as a signature of creativity, and from this viewpoint, the ST and SW networks are most supportive of creativity.

\section{DISCUSSION}

The combination of recurrent connectivity, competitive activity and modulation creates a rich dynamics in all the model networks. The purpose of this study was to see what difference the type of connectivity made to the emergence of metastable patterns of activity, since these are seen as corresponding to the significant cognitive representations (ideas) implicit in the system.

The results shown in Figures 4 - 7 lead to two important observations:

1) ST connectivity, which is both small-world and power law, generates a large number of distinct metastable patterns which persist for a moderate duration, whereas LO and SW connectivity lead to a smaller number of distinct patterns which are generated repeatedly and persist somewhat longer. Thus, the dynamics of the latter two networks is not as rich as that of the ST network, even though the amount of time spent in metastable states is higher for LO and SW networks. Since the SW and ST networks have the same degree of clustering (and LO much higher clustering), the results suggest that the richer dynamics is a consequence of the power law aspect of connectivity in the ST network. On the other hand, the RA network, which has a degree distribution similar to the SW and LO networks, generates a higher number of metastable patterns than either of these, but fewer than the ST network.

2) The internal coherence of the metastable patterns generated by the networks is directly proportional to the clustering in their connectivity. This is not surprising, but does show the value of structural clustering in confining the dynamics to generating more "meaningful" patterns.

From these two observations, we conclude that the ST network represents an elegant way to obtain the primary advantage of clustered connectivity - coherence - without the associated loss of richness in the dynamics. We speculate that clustering tends to confine the dynamics to localized regions of activity space, leading to repetitions. The existence of high degree nodes implied by the power law connectivity counters this tendency by creating more options for the dynamics at each step. This results in a more "tangled" dynamics, and a richer sampling of metastable states. Thus, ST networks may be a neural instantiation of Mednick's deep associative hierarchies. A detailed investigation of this is left to future studies.

\section{Conclusions}

In this study we systematically investigated the effect of connectivity on the dynamics of a neural network model of semantic search in the space of conceptual combinations. The study showed that networks with clustered connectivity and non-fat tailed degree distributions are prone to generating repeated but internally coherent metastable activity patterns, while networks with unclustered connectivity generate more diverse but less coherent patterns. A network with smallworld connectivity and power-law degree distribution is able to combine the advantages of both while avoiding their disadvantages. This type of connectivity has been observed empirically in real-world semantic networks [16]. This provides an intriguing correspondence between semantic organization and semantic richness, raising the question of whether the observed semantic organization has evolved to support richer semantic possibilities.

\section{ACKNOWLEDGMENT}

The authors would like to thank Laxmi Iyer, Vince Brown, Simona Doboli, Paul Paulus and Dan Levine for useful discussions and ideas. 


\section{REFERENCES}

[1] D. Campbell, "Blind variation and selective retention in creative thought as in other knowledge processes," Psychol. Rev., vol. 67, pp. 380-400, 1960.

[2] S. Mednick, "The associative basis of the creative process," Psychological Review, vol. 69(3), pp. 220-232, 1962.

[3] V. Brown, M. Tumeo, T. Larey, and P. Paulus, "Modeling cognitive interactions during group brainstorming," Small Group Research, vol. 29, pp. 495-526, 1998.

[4] D. Simonton, "Scientific creativity as constrained stochastic behavior: the integration of product, person, and process perspectives," Psychol. Bull., vol. 129, pp. 475-494, 2003.

[5] B. Nijstad and W. Stroebe, "How the group affects the mind: A cognitive model of idea generation in groups," Personality and Social Psychology Review, vol. 3, pp. 186-213, 2006.

[6] M. Schilling, "A small-world network model of cognitive insight," Creativity Res. J., vol. 17, pp. 131-154, 2005.

[7] H. Poincar, The Foundations of Science. Science Press, 1921.

[8] J. March, "Exploration and exploitation in organizational learning," Organization Science, vol. 2, pp. 71-87, 1991.

[9] C. Nemeth, "Dissent as driving cognition, attitudes, and judgments," Social Cognition, vol. 13, pp. 273-291, 1995.

[10] J. Raaijmakers and R. Shiffrin, "Search of associative memory," Psychological review, vol. 88, 1981.

[11] G. McKoon and R. Ratcliff, "Spreading activation versus compound cue accounts of priming: Mediated priming revisited," Journal of Experimental Psychology: Learning, Memory, and Cognition, vol. 18, pp. 1155-1172, 1992.

[12] R. Ratcliff and G. McKoon, "Retrieving information from memory: Spreading-activation theories versus compound-cue theories," Psychological Review, vol. 101, pp. 177-184, 1994.

[13] D. Nelson, D. Bennett, N. Gee, T. Schreiber, and V. McKinney, "Implicit memory: Effects of network size and interconnectivity on cued recall," Journal of Experimental Psychology: Learning, Memory, and Cognition, vol. 19, pp. 747-764, 1993.

[14] D. Nelson and J. Xu, "Effects of implicit memory on explicit recall: Set size and word frequency effects," Psychological Research, vol. 57, pp. 203-214, 1995.

[15] D. Nelson, T. Schreiber, and C. McEvoy, "Processing implicit and explicit representations," Psychological Review, vol. 99, pp. 322-348, 1992.

[16] M. Steyvers and J. Tenenbaum, "The large scale structure of semantic networks: Statistical analyses and a model of semantic growth," Cognitive Science, vol. 29, pp. 41-78, 2005.

[17] A.-L. Barabasi and R. Albert, "Emergence of scaling in random networks," Science, vol. 286, pp. 509-511, 1999.

[18] R. Albert and A.-L. Barabasi, "Statistical mechanics of complex networks," Reviews of Modern Physics, vol. 74, pp. 47-97, 2002.

[19] M. Newman, S. Strogatz, and D. Watts, "Random graphs with arbitrary degree distribution and their applications," Physical Review E, vol. 64, p. 026118,2001

[20] H. Yook, S.-H. ad Jeong and A.-L. Barabasi, "Modeling the internet's large-scale topology," Proceedings of the National Academy of Sciences USA, vol. 99, pp. 13 382-13 386, 2002.

[21] M. Newman, Networks: An Introduction. Oxford University Press, 2010 .

[22] D. Watts and S. Strogatz, "Collective dynamics of "small-world" networks," Nature, vol. 393, pp. 440-442, 1998.

[23] D. Watts, Small Worlds: The Dynamics of Networks between Order and Randomness. New Jersey: Princeton University Press, 1999.

[24] J. Bohland and A. Minai, "Efficient associative memory using smallworld architecture," Neurocomputing, vol. 38-40, pp. 489-496, 2001.

[25] O. Sporns and J. Zwi, "The small world of the cerebral cortex," Neuroinformatics, vol. 2, pp. 145-162, 2004.

[26] L. Iyer, A. Minai, S. Doboli, V. Brown, and P. Paulus, "Effects of relevant and irrelevant primes on idea generation: A computational model," in Proceedings of IJCNN 2009, 2009, pp. 1380-1387.

[27] L. Iyer, S. Doboli, A. Minai, V. Brown, D. Levine, and P. Paulus, "Neural dynamics of idea generation and the effects of priming," Neural Networks, vol. 22, pp. 674-686, 2009.

[28] L. Iyer, V. Venkatesan, and A. Minai, "Neurcognitive spotlights:configuring domains for ideation," in Proceedings of WCCI 2010, 2010, pp. 3026-3033.
[29] R. Zucker and W. Regehr, "Short-term synaptic plasticity," Annual Review of Physiology, vol. 64, pp. 355-405, 2002.

[30] L. Abbott and W. Regehr, "Synaptic computation," Nature, vol. 431, pp. 796-803, 2004

[31] I. Tsuda, "Towards an interpretation of dynamic neural activity in terms of chaotic dynamical systems," Behavioral and Brain Sciences, vol. 24, pp. 793-847, 2001.

[32] R. Eckhorn, R. Bauer, W. Jordan, M. Brosch, W. Kruse, M. Munk, and H. J. Reitboeck, "Coherent oscillations: A mechanism of feature linking in the visual cortex?" Biological Cybernetics, vol. 60, pp. 121130, 1988.

[33] W. Singer and C. M. Gray, "Visual feature integration and the temporal correlation hypothesis," Annual Reviews of Neuroscience, vol. 18, pp. 555-586, 1995.

[34] W. Singer, A. Engel, A. Kreiter, M. Munk, S. Neuenschwander, and P. Roelfsema, "Neuronal assemblies: Necessity, signature and detectability," Trends in Cognitive Sciences, vol. 1, pp. 252-260, 1997.

[35] A. Engel, P. Fries, and W. Singer, "Dynamic predictions: oscillations and synchrony in top-down processing," Nature Reviews Neuroscience, vol. 2, pp. 704-716, 2001.

[36] F. Varela, J.-P. Lachaux, E. Rodriguez, and J. Martinerie, "The brainweb: phase synchronization and large-scale integration," Nature Reviews Neuroscience, vol. 2, pp. 229-239, 2001.

[37] S. Bressler and E. Tognoli, "Operational principles of neurocognitive networks," International Journal of Psychophysiology, vol. 60, pp. 139-148, 2006.

[38] D. L. Wang and D. Terman, "Locally excitatory globally inhibitory oscillatory networks," IEEE Transactions on Neural Networks, vol. 6 , pp. 283-286, 1995. 